Training in Evidence-Based Practice across the Professional Continuum 


\begin{abstract}
The benefits of evidence-based practice (EBP) and the utilization of evidence-based treatments (EBTs) is well documented in the professional practice and training literature.

However, the literature explaining methods by which to train and supervise professionals in these areas is less clear. To facilitate the highest level of clinical care, mental health trainee, professionals, and those who educate and supervise clinicians will benefit from identifying and evaluating effective and efficient methods for implementing EBP and EBTs. This manuscript reports on three programs across the training and professional continuum that provide training and supervision/consultation on EBT. Commonalities among the program include an individualized training approach, the use of active learning strategies, and measuring individuals' progress in learning and/or outcomes. Data regarding student response to EBP and EBT training in graduate study is provided. Additionally, a model for internship training in clinical research and clinical practice is described. Finally, pilot data is provided regarding training methods for preparing professional clinicians to provide an EBT. The development of models addressing EBP and EBT in academic, training and professional practice areas is instrumental in developing skilled clinicians and researchers able to address the clinical needs of their clients.
\end{abstract}

Search Terms:

Training, Evidence-based practice, Evidence-based treatment, Graduate psychology, Internship 
Evidence-based practice (EBP) is central to health care delivery (Institute of Medicine, 2001; Lehman, Goldman, Dixon, and Chruchill, 2004), and the implementation of evidencebased treatments (EBTs) is an integral part of this process. However, concerns exist related to barriers impacting training, dissemination, and supervision of these interventions (Connor-Smith \& Weisz, 2003; Essock et al., 2003; Rakovshik \& McManus, 2010; Stewart \& Chambless, 2007; Weissman et al.; 2006). Given the need for EBP, it is paramount that psychologists and mental health practitioners receive exposure to, training on, and supervision in ETBs across the professional continuum.

The research to support that EBP is useful in the remediation of a wide variety of symptoms and clinical disorders among adults and youth is growing (Hunsley \& Lee, 2007). Therefore, it is important to ensure that training across the profession of psychology and behavioral health includes a focus on proficiency in delivering EBP that includes EBTs. Dosage of training in EBP, such as cognitive-behavioral therapy, directly relates to therapist competence and patient outcomes (Rakovshik \& McManus, 2010). The clinical child psychology field specifically, and clinical psychology more generally, have indicated that EBP should be the cornerstone of any training model for clinical psychologists. For example, in 2009, APA sponsored a Summit on the Future of Psychology in San Antonio, TX where 150 delegates (i.e., division representatives, governance groups, primary care administrators) met to discuss how mental health practice has evolved and one component of this discussion was the need to establish training guidelines that include evaluating and disseminating evidence for the services they provide.

Exemplary models for training in EBP and EBTs more broadly are generally lacking. Due to difficulties transporting these interventions, recent guidelines have been developed for 
training practitioners in the use of EBTs emphasize the importance of specialized techniques designed to engage, teach, and support practitioners in their use of EBT skills (Hyde, Falls, Morris \& Schoenwald, 2003). Barriers to the use of EBP in training environments include financial and operational constraints, clinic/program support, client population needs, supervision and consultation capacities, and length of treatment. Across the wide spectrum of psychotherapy providers, many professionals matriculate from doctoral and master's level training programs without receiving sufficient training in implementing specific EBP techniques and skills (Weissman et al., 2006).

The goal of this paper is to describe current efforts to address training in evidence-based practice with a focus on evidence-based treatments across the professional practice continuum using examples from clinical child graduate and pre-doctoral internship training, along with professional practice.

\section{Graduate-level training}

Clinical child psychology training programs have a responsibility to ensure that didactic instruction and practicum training provide the basis for competency in EBP. Although accreditation guidelines prompt programs to provide and document their efforts to teach EBP, little is known about how programs should accomplish this goal. The field would benefit from some examples to inform a dialog about which practices, courses, policies, and efforts are producing effective clinical child trainees. In that vein, what follows is an illustration of how EBP is taught at the University of Kansas Clinical Child Psychology Program (KUCCPP). The KUCCPP approach to EBP training is to infuse the value of evidence throughout the clinical curriculum by providing awareness, knowledge, and practice to its students. 
The training program at KU is based on the Boulder Model; a model that requires that science be applied to all training efforts One key difference in training approach between EBPbased and non-EBP-based training programs is the inclusion of formal assessment of client change and student learning outcomes.

Across courses and practicum experiences, the use of EBP at the KUCCPP is structured around the following tenants: a) use of published manualized treatments, b) discussion of what behaviors by therapist or activities in session have an evidence-base, c) discussion of reasonable modifications to the treatment protocol (i.e., cultural adaptations or adaptations based on student's level of skill), and d) evaluation of the student's application of the approach (selfevaluation).

\section{Coursework and practicum expectations}

Didactic instruction is the foundational approach used for learning later clinical skills in graduate school. In the first year of graduate training, students at the KUCCPP take courses in developmental psychopathology, psychological assessment, and attend a practicum team. This coursework lays the base for learning in practicum where junior students shadow advanced students demonstrating clinical skills with clients. Attending practicum team also allows for firstyear students to discuss EBP in a weekly group format. Additionally, they are responsible for reviewing the treatment approach, reading the relevant literature that provides the evidence for the treatment used, and suggesting several questions for consideration by the primary therapist. The approach at KU is consistent with the Association of Directors of Psychology Training Clinics (ADPTC) Competencies Workgroup report, in which the ADPTC suggested that coursework prior or concurrent to direct practicum experience should provide "i) knowledge of scientific, theoretical, empirical, and contextual bases for intervention; ii) training in basic 
professional skills, such as empathic listening, framing of problems, and so forth; and iii) training in assessment of treatment progress and outcome" (ADPTC Practicum Competencies Workgroup, 2004, p. 7).

In the second year, students take courses that introduce them directly to the principles of treatment (i.e., clinical interviewing, active listening) and it is in their second year of practicum that students learn specific EBT methods and protocols (i.e., Parent-Child Interaction Therapy and Trauma-focused Cognitive Behavioral Treatment) via weekly review with their supervision team of the weekly session goals and materials from a given protocol. At the same time, students take courses on diagnostic assessment, clinical intervention with children and families, and advanced practicum, which involves treating clients in the KU clinic. Expectations for beginning therapists are outlined in the clinic manual as well as during practicum team meetings. Students beyond their second year in training continue to take advanced practicum courses and attend supervision team meetings composed of a vertical team of students in their first, second, and higher years in the program. The use of a vertical team allows for discussion of the basics as well as the complexities in how EBP and EBTs are implemented across a range of clinical cases and experience levels. Evaluation is primarily accomplished via weekly discussion between students and practicum supervisors about the progression of treatment and by formal evaluation of the students' performance at the end of each semester.

\section{Tools for training}

The KUCCPP has a training clinic where student therapists see clients from the local community and larger metropolitan area. As a part of the training mission of the clinic, treatment manuals are purchased, updated and provided for use by the student clinicians. Additionally, the clinic does not ascribe to any one treatment approach or manualized treatment for any one 
disorder, but instead includes a range of EBTs from which students can choose. Students are encouraged to discuss their choices with their supervisor and think critically about why they chose the approach, why the approach might work best for a particular client, and how the intervention will be evaluated and modified if necessary.

In consultation with the student, supervisors also assess the students' readiness to learn new approaches by documenting the students' progress with one EBT. Further, the supervisor determines if the student understands the components of the approach, is able to administer the approach consistent with the treatment manual, and if the student is able to make modifications to implementation when needed to meet the needs of the client. Students are also questioned regularly in supervision to ascertain their ability to think critically about the approach used and encouraged to think about indicators of success and failure of the intervention. The most common assessment method is the review of the recorded session videotape. Supervisors and trainees review tapes together allowing for supervisors to point out where implementation was and was not effective and encourage the trainee to do the same. Because the more formal evaluation of the student's knowledge of EBP is conducted in the coursework, practicum supervision is viewed as a time to evaluate the implementation, the students' ability to actually use and administer the treatment.

\section{Documentation of competency}

All students in the KUCCPP are evaluated, formally and informally in their ability to choose, implement, and evaluate their use of EBP. The primary formal way this is done is via supervisor evaluation at the end of each practicum semester. Measurement of competency in EBP is an important component of student development. To this end, students and supervisors complete an evaluation form that compares the students' performance to expected benchmarks 
for a given year of training across several domains. Specifically, students are evaluated in terms of their professional conduct, therapy skills, assessment skills, use and understanding of diversity, written reports, and use of supervision. For each area, supervisors indicate if the student is performing as expected for their year in the program using pre-designated indicators of progress. The indicators of progress and performance tool used by KUCCPP was informally developed. Specifically, the clinic director asked the director of other training clinics for the tools they use to evaluate their students. The clinic supervisors at KU reviewed the practicum evaluation tools from other training clinics and created a new tool based on a consensus across supervisors as to which items the supervisors felt represented the most thorough and useful items for evaluating clinic performance. Students are informed of their clinical strengths as well as areas that need improvement. Students also complete an evaluation form that consists of their self-ratings of their clinical skills, improvements made over the semester, and areas that require additional training.

Example evaluation of EBP: Data on KU CCPP trainees

Although the training model for the KUCCPP provides for learning of EBP, data is required to identify if the goal of training actually translates to the students. In spring 2011, the 20 student therapists at the KU Child and Family Services Clinic were surveyed to determine their use and understanding of training in EBP for both treatment and assessment clients. The purpose of this assessment was not to evaluate treatment fidelity in any formal sense, but instead served as an exercise for students to think about what they do in treatment and how they understand the role of EBP.

When asked about the use of EBP in treatment cases, 52.6\% reported always using EBP. For those students who endorsed not always using an evidenced-based approach to treatment, the 
most common reason given was because the referral issue did not neatly fit a manualized treatment protocol. A similar result was found for use of EBP in assessment cases (57.9\%). For students who endorsed at times not using EBP in assessment cases, the most common reason given was a sense of confusion about what EBP for assessment looks like. This latter finding was interesting and a follow-up question provided some insight as to why students might be confused about their use of EBP. When asked how they know if what they are doing is in line with EBP, students indicated that they know the approach is evidenced-based if "the information is in a manual and the treatment has been studied through efficacy or effectiveness research, if their supervisor told them if was evidenced-based, or if they had read a review article or other materials documenting the evidence for a given treatment." It is important to note that students also reported a general sense that EBP is linked to a disorder or a specific referral question and that when clients who do not meet criteria for a disorder, the use or approach to EBP was less clear.

Students were also asked which EBTs they felt competent to use and how they evaluate their use of EBP. The majority of students indicated feeling competent to use programs like Defiant Child or Coping Cat, or Parent-Child Interaction Therapy, (90\%, 87\%, and 66\% respectively). In terms of evaluating their use of EBTs, students indicated that they use multiple methods to determine if the treatment is effective. Seventy-nine percent indicated that they rely on supervisor feedback to know if they are using the approach correctly. The next most common method ( $68 \%$ of the sample) reported using feedback from the client that the target behavior had improved or using another measure (i.e., behavior checklist) to document symptom improvement during and post-treatment. Perhaps surprisingly, almost half of the students indicated that review of their therapy tapes was one of the methods used to track their progress using EBP. Although 
self-evaluation is not a part of a formal course, it appears that students have learned the value of self-review as a part of EBP implementation. Reviewing of tapes is an important component of the active learning process valued by the program.

The information from the survey of students provided the program with interesting feeback about how students view their use of EBP. Although this simple survey is no substitute for a proper evaluation of treatment fidelity, it does provide some important information about how well the training efforts to integrate clinical science may be working. The next step will be to provide not only formal fidelity tests as suggested by Rakovshik and McManus (2010), but also evaluate how well the training on EBP actually translates into good outcomes for clients.

A primary goal of the KUCCPP graduate training model is that, as students matriculate through the program, their foundation for learning and thinking critically about evidence will provide them the proper foundation for demonstrating their EBP competencies while on internship.

\section{Training on Internship}

The internship year is traditionally looked upon as a year for intensive clinical training, broadening clinical experiences and expertise, and amassing clinical hours necessary for licensure. With the current emphasis in the field on training in evidence-based approaches to assessment and intervention, the extent to which internship sites provide adequate training in the accumulation and use of research evidence in delivering treatment has become an issue of interest to the field. The purpose of this section is to describe one model of integrating clinical research and clinical practice in internship training in order to facilitate interns' understanding of how the creation and use of evidence supports the development and provision of effective services. 


\section{University of Illinois at Chicago Department of Psychiatry: Clinical-Scientist Internship}

\section{$\underline{\text { Training }}$}

One method of ensuring that interns receive adequate training in the provision of evidence-based interventions is to incorporate clinical research experiences into the internship training year. The Academy of Psychological Clinical Science (APCS) is a consortium of graduate programs and internship sites that are committed to providing graduate students in psychology with training in clinical science and evidence-based practice. The internship program in the Department of Psychiatry at the University of Illinois at Chicago (UIC) ascribes to this model of training and aims to provide an internship program where interns receive mentored clinical research experience integrated with clinical practice. The UIC program is guided by three principles: (1) the integration of clinical research and practice; (2) training in effective interventions for under-served populations; and (3) intensive mentorship in clinical practice and clinical research from faculty who ascribe to the clinical scientist model.

\section{Principle \#1: Integration of clinical research and practice}

The first set of goals related to this principle is to train psychologists who are capable of: a) implementing empirically-supported mental health services; b) evaluating the effectiveness of existing treatments; and c) developing innovative treatments based upon existing and emerging empirical findings. Consistent with these goals and with the values of clinical science programs, we expect graduates of our internship to function as clinical investigators and/or as practitioners who utilize scientific methods in professional practice. The second goal related to this principal is to provide instruction and experience in scientific methods and data so as to develop interns' competencies to apply scientific principles in practice. This goal is operationalized through formal courses and seminars in scientific methods for clinical research, as well as through 
mentored clinical research experiences on faculty projects. The third goal for this principle is for interns to develop competencies in the development and implementation of clinical practice including assessment and evaluation, consultation, individual therapy, group therapy, and family therapy, and in clinical research programs that advance new treatment modalities across these domains.

\section{Principle \#2: Effective interventions for under-served populations}

The goal for this principle is the development of competencies in the evaluation and implementation of effective interventions for urban, low income, minority adults, children, and families. Experience conducting research and clinical practice with this target population provides interns with opportunities to develop an integrated understanding of the influence of ethnicity, culture, socioeconomic status, and poverty on client functioning, and to understand how clinical research informs the provision of services that effectively address diversity in these factors.

\section{Principle \#3: Faculty mentoring}

The first goal for this principle is for interns to receive intensive supervision in clinical practice and clinical research from faculty who conform to the clinical science model (i.e. faculty who are clinical researchers or practitioners who utilize research findings to guide clinical practice or generate clinical theory that can guide research). The second goal is for interns to collaborate with faculty in the development of clinical research projects to enhance their development as independent clinical researchers.

These training principles and goals are achieved through individualized training programs. Each intern designs his/her own individual program under the guidance and mentorship of the training directors based on individual training needs and career goals, and with 
a particular concentration in mind. Interns choose from a menu of clinical research and clinical practice experiences. For example, an intern may choose the following curriculum consistent with a child prevention and intervention concentration: a 12-month rotation on a delinquency prevention research project, a 12-month rotation on a school-based services research project, a 6month rotation studying biostatistics and analyzing secondary data with faculty mentor, a 12month rotation through the Disruptive Behavior Disorder clinic, a 12-month rotation through the Childhood Stress and Anxiety Clinic, and a 6-month rotation through the Pediatric Mood Disorders Clinic. Rotations can be 6 or 12 months and interns typically spend $10-20 \%$ of their time on each rotation in order to allow them to obtain a range of experiences. Interns' participation and ability to meet the goals and objectives of each rotation and the overall internship is regularly evaluated by their supervisors and the training director throughout the year. Supervisors provide feedback and opportunities for frequent exchange to ensure that interns are achieving the internship's goals to train in clinical research and evidence-based intervention, as well as achieving their own goals for training and career development.

\section{An example: Clinical Practice and Research Training in Pediatric Mood Disorders at UIC}

Each rotation strives to create an experience consistent with the clinical science goals of training. Thus, within each rotation faculty members aim to present interns with integrated clinical and research experiences that facilitate the development of good clinical scientists through the provision of EBP and participation in the creation of evidence to contribute to science-based practice.

One of the rotations offered at UIC is the Pediatric Mood Disorders Clinic (PMDC). The PMDC is an integrated clinical research program within the Division of Child Psychiatry. Clinicians in the clinic conduct psychiatric evaluations and deliver individual, family, and group 
treatment based on our child and family-focused cognitive-behavioral treatment (CFF-CBT)

model. CFF-CBT is a 12 session manual-based family-focused psychotherapy for youth 7-13 with bipolar disorder that integrates cognitive-behavioral principles with psycho-education, interpersonal psychotherapy, mindfulness, and positive psychology. This treatment model has shown promise in several open trials and is currently being tested in a randomized clinical trial (West \& Pavuluri, 2009; West, et al., 2009).

The training goals of this rotation include: (1) clinical training in the treatment of childhood mood disorders through supervised clinical evaluation and treatment; (2) training in EBT for pediatric bipolar disorder (CFF-CBT) and supervision in the delivery of this treatment as part of a clinical research trial; and (3) an understanding of the development of an evidence base for the treatment of pediatric mood disorders through participation in clinical research. Interns can choose from various integrated clinical and clinical research experiences to meet these goals. Opportunities include: a) carrying a caseload of individual/family therapy cases (youth 0-18 with mood disorder spectrum disorders); b) receiving training in CFFCBT/RAINBOW therapy, including training on the manual, delivering the treatment as part of clinical trial, and conducting baseline and follow-up assessments as part of the clinical trial; c) participating as a co-leader in our RAINBOW group therapy program (adapted group format of treatment model); d) conducting data analyses; e) receiving supervision; and f) participating in didactics, case seminars, and team meetings. Interns may choose to structure their rotation based on their own training goals. For example, an intern may choose an integrated clinical/clinical research rotation, for $20 \%$ time over 12 months. Table 1 illustrates an example rotation structure.

The UIC internship model represents one example of an attempt to train clinical scientists through individualized, yet structured integrated clinical and clinical research experiences and to 
prepare psychologists to utilize effective scientific principles in their clinical research and practice. Hopefully, with increased attention to training psychologists in clinical science and evidence-based practice, other models for achieving these aims will be implemented, tested, and disseminated to ensure that, across the discipline, programs are generating well-trained professionals who will make important contributions to creating and practicing effective treatments.

\section{Training Professional Clinicians}

Graduate and training programs have begun to implement formal methods for EBP and EBT training and institutions will need to be equipped to continue this exposure and training for their staff. However, models for ongoing training in EBTs after formal training has occurred and psychologists have entered the professional workforce are less well-articulated (Beidas, \& Kendall, 2010).

Expectations and support for implementing EBTs in clinical practice are well indicated. However, the approach and mechanisms for training and implementing these interventions are less clear and formal. To address the need for an organized and empirical approach to training in EBTs at the professional practice level and to continue to meet the clinical needs of a diverse client population, staff at Nationwide Children's Hospital Behavioral Health Services (NCH BHS) began an effort to increase professional clinicians exposure, knowledge, training, and implementation of EBTs being cognizant of implementation strategies and barriers (Gotham, 2006). One specific intervention that has been implemented through this endeavor is MultiFamily Psychoeducation Psychotherapy (MF-PEP) (Fristad, Goldberg-Arnold, \& Leffler, 2011). MF-PEP is a manualized eight-week adjunctive group therapy for children ages eight to twelve with mood disorders and their caregivers. Professionals trained in this intervention through the 
$\mathrm{NCH}$ BHS program have included psychologists, social workers and counselors as well as trainees from various disciplines. Similar to other programs that have focused on training and utilizing EBTs (Stirman et al., 2010) NCH BHS staff have developed and piloted three training models that incorporate increased knowledge of the intervention, along with supervision and consultation on the application of the techniques. Currently the three models which were developed in response to the varying clinical needs of the clinicians, serve as a training approach for EBTs across the professional continuum. The goal of training is to build on existing knowledge and skills sets that a professional clinician may have been exposed to or developed during graduate school and previous clinical placements. The models also address training professional clinicians who may not have had previous exposure to EBT or similar manualized interventions. An overview of the development of the training models and consultation components is provided below, as well as clinician and trainee feedback on the training methods.

\section{Clinic Setting}

$\mathrm{NCH}$ BHS provides a variety of outpatient mental health services across four outpatient clinics throughout the Columbus area. The clinical population is representative of typical youth mental health needs. NCH BHS employs approximately 70 professional clinicians (i.e., psychologist, social workers, and counselors). Additionally, on average three predoctoral psychology interns, three postdoctoral psychology fellows, and five social work graduate students are trained each year.

\section{Multi-Family Psychoeducation Psychotherapy}

\section{Intervention}

MF-PEP is an adjunctive treatment for youth with mood disorders and their caregivers that provides psychoeducation, support, and skill development related to mood disorders 
(Fristad, et al., 2011). Efficacy studies have found MF-PEP to be successful at changing consumers attitudes toward treatment, increasing knowledge of mood disorders, improving family functioning, and decreasing mood severity (Fristad, Goldberg-Arnold, \& Gavazzi, 2002; Fristad, Verducci, Walters, \& Young, 2009). Currently, the transportability of MF-PEP to a community mental health setting is being evaluated (MacPherson, Leffler, \& Fristad, 2012).

\section{Training}

To train professional clinicians in MF-PEP, NCH BHS has piloted three training models. The models consisted of the original (5 hours), broad (7 hours), and focused (4 hours) approaches. Table 2 presents detailed information about each training model. The topics addressed in the training approaches were similar but differed in the extent of content covered. For instance, in the original training an overview of the research protocol and aims was not provided due to this occurring in a separate meeting with clinicians involved in the research protocol. Additionally, as clinicians were able to view active groups at NCH BHS the need to incorporate video clips of the group in training no longer existed.

Professional clinicians received one of the three training models. In addition, they received one hour of consultation a week while co-facilitating MF-PEP for the first time. After that, they received a minimum of 30 minutes of consultation and utilized consultation as needed that was initiated by the professional clinician or MF-PEP consultant. Feedback on skills and competence was provided informally during consultation sessions by a MF-PEP consultant.

Psychology trainees received the focused training model. In addition, they received four hours of didactics focused on group therapy and mood disorders in youth. While co-facilitating the intervention they received one hour of individual supervision a week and additional supervision on the intervention and relevant topics as needed or requested. 
$\mathrm{NCH}$ BHS has trained 28 individuals across the professional continuum. In the original approach one licensed psychologist and six licensed social workers were trained. The Broad approach was used to train two licensed psychologists, four licensed counselors, six psychology interns and three psychology postdoctoral fellows. The focused approach was implemented to train one licensed psychologist and five licensed social workers. Of the professional clinicians trained $33 \%$ had been practicing for 12 or more years, $33 \%$ had practiced for five to eight years, $25 \%$ had practiced for one to four years, and $9 \%$ had practiced for less than one year.

\section{$\underline{\text { Results of Training by Approach }}$}

Twenty-three clinicians trained in MF-PEP completed the MF-PEP Clinician Feedback Post (MF-PEP CF-P) survey after they facilitated their first group. The MF-PEP CF-P is a 22item post-group survey addressing elements of the training received, experience facilitating the group, and demographic areas. Five clinicians did not complete the survey due to not having facilitated a MF-PEP group. Selected items from that survey using a Yes/No format $(1=$ Yes, $2=$ No) addressing training experience are presented in Table 3. Overall, those that received the focused approach reported feeling the least prepared to facilitate MF-PEP. Additionally, based on informant feedback this method was the one most lacking in skills/topics related to the group. Narrative responses for the lack of training on specific skills/topics in the focused method consisted of wanting to see a live group before facilitating the intervention ( 3 respondents), being provided a better explanation of the ongoing supervision/consultation with the option for processing group and receiving direct feedback of skills (2 respondents), and having more training on specific topics (e.g. specific group activities, weekly projects, screening for group, and recruitment) (1 respondent). 
Forty-two percent of those who received MF-PEP training had prior training on a manualized intervention. Thirty-three percent of staff in the original training method had previous training on a manualized treatment compared to $100 \%$ of staff in broad training method. Additionally, a majority of staff in the focused training method had received prior training on manualized interventions.

\section{Future goals for the NCH BH MF-PEP program}

In responding to clinician feedback regarding observing sessions, MF-PEP developers and consultants at NCH BHS are currently discussing the feasibility of observing MF-PEP sessions prior to training. Efforts have been made to address other areas of training concern. For example, more time is spent explaining the supervision/consultation process and providing reminders to professional clinicians about the use of consultation. Two meetings occur during the year where MF-PEP trained clinicians can discuss group process and consultation concerns in a group format. Additionally, more detailed information is provided regarding recruitment. A three hour screening and recruitment training separate from the one-day training has been implemented.

Regarding fidelity checks and formal feedback, a system employing newly developed measures for fidelity ratings and feedback is being developed. These measures along with feedback regarding the clinician's overall group therapy skills will be implemented.

Feedback has supported the ongoing use of the broad model of training. The implementation of the brief model will be considered as either a quick model to refresh skills and knowledge after not facilitating the intervention for an extended period, or if fidelity ratings begin to decline. 
Results of this survey also suggest that trainees may have a prior knowledge and skill set with utilizing a manualized treatment compared to their professionally licensed colleagues. Given this background, likely gained through their graduate training and previous clinical placements, training efforts may not need to focus heavily on topics such as flexibility with fidelity (Kendall, Gosch, Furr, \& Sood, 2008).

It appears that trainees entering the workforce may have had more exposure to EBTs or manualized interventions compared to their licensed colleagues. This is consistent with the ongoing shift in graduate training programs providing more training and supervision in this area as well as internship programs offering training on EBTs.

\section{Conclusion}

The vast majority of the required supervised clinical training in psychotherapy across programs training psychotherapists is in non-evidence based practices, and the obstacles that exist with regard to training in EBP are numerous, and include lack of trainee interest, lack of qualified faculty supervisors, time commitment to learn EBP, and difficulty in teaching (Weissman et al., 2006). Nonetheless, these barriers are not insurmountable, and the field of Clinical Psychology has been at the forefront of the mental health professional workforce in providing training to deliver EBP and EBTs. This paper describes several training models that have been developed to enhance skills and techniques of EBPs across the professional continuum. Together, these training models provide some common elements that are useful to consider in light of current goals to increase the number of psychologists and other mental health professionals who are trained to use EBP approaches.

The first common element among the models is that training in EBP can be approached in a flexible, individualized way that is tailored to meet the needs of the trainee and the client. The model used at KUCCPP, whereby the student therapist is allowed the opportunity to select a 
treatment manual or intervention materials based on the presentation and needs of the client, and to adapt the protocol if necessary, serves as a useful paradigm that could be replicated by other training clinics. Likewise, constructing a personalized internship experience with rotations chosen by the trainee to meet their professional goals and needs, as described in the UIC model, provides for a meaningful and relevant experience where areas of focus can be expanded and gaps in training can be addressed. The concept of "graded training" whereby greater instruction is offered to therapists who are slow to develop competence has been described by Rakovshik \& McManus (2010) as a potentially efficient way to train heterogeneous trainees.

A second theme that emerges, and is consistent with the broader literature (Beidas \& Kendall, 2010; Rakovshik \& McManus, 2010), is that training is particularly effective when it involves active learning components. Reading, self-directed trainings, and workshops alone do not consistently result in significant changes in skills or treatment mastery (Herschell, Kolko, Baumann, \& Davis, 2010). As the data from Nationwide Children's Hospital indicates, there is a risk to streamlining training, even for licensed professionals, to only the barest of essentials. Half of the therapists who received the briefest (i.e., focused) training on MF-PEP did not feel prepared to run groups, and they exited the training with the desire to observe a live group and to receive more direct feedback on their skills prior to beginning the clinical intervention. Although the sample size is too small for statistical comparison, the staff who received an additional 1-3 hours of training, generally involving more time explaining and demonstrating the complete treatment protocol and ways to implement it rather than one part, were incrementally more likely to feel prepared. Active learning is achieved at KUCCPP in vertical teams of students at different years of training that allows for skills and knowledge to be gained at multiple levels, from the more novice learner who may be attuned to general ways to structure the session, to an 
experienced learner who considers nuances in verbal and nonverbal communication. Likewise, at UIC, interns participate in mentored clinical research experiences so they are actively involved in generating data to evaluate evidence for interventions, in addition to learning and applying the interventions themselves. This approach facilitates critical thinking and an in-depth understanding of how an evidence base for an intervention is actually evaluated. Each of these models is consistent with research suggesting that active learning strategies (e.g., behavioral role-plays, coaching, experimental exercise) are necessary in addition to change therapist behavior (Beidas \& Kendall, 2010; Herschell et al., 2010).

Third, training in EBP by nature involves measurement across time and across people. The assessment of the client's presenting problem at the start of treatment, and measures of symptoms, functioning, or improvement throughout the therapy process to track changes over time is particularly critical. As shown at KUCCPP, one common way that students evaluate their use of EBP is to track client improvement. In addition to client ratings, there are regular mechanisms to document trainee competency in the form of self-ratings, supervisor ratings, and qualitative feedback. Supervisor feedback was the most important mechanism listed by trainees to evaluate their use of EBP in the Kansas program. At UIC, the involvement in clinical work and research experiences are complementary, so that interns can observe the interplay between clinical trials, clinical care, and measuring outcomes. Interns are actively involved in clinical research focused on using rigorous research designs to assess efficacy, measure key outcomes, and test fidelity in the delivery of interventions as part of clinical research and practice. In addition, interns' ability to achieve the internship's objectives for training in clinical research and evidence-based intervention delivery are evaluated by their supervisors and the training director throughout the year so that interns receive concrete feedback regarding their strengths and 
weaknesses. Interns and supervisors are able to work together to enhance interns' training experiences and their ability to successfully master the desired skills. Finally, at NCH BH, there is systematic evaluation of trainees following training in EBTs that has helped to shape decisions about how much training and what components are most important to achieve competence.

Limitations of this manuscript include its focus on three specific programs amidst the hundreds of graduate, internship, and professional training programs in existence, as well as lack of specific information about how to actually replicate these models in other locations. However, our hope is to highlight models that could serve as guides for transforming standards in the field to include a more prominent focus on training in EBP and EBTs. Obviously, there is tremendous diversity across sites that would need to be considered in applying models such as these to meet the needs and circumstances of a particular training environment.

Further information about these programs can be acquired by contacting the authors of this manuscript as well as accessing each program's website. Overall, we hope that the articulation of these three training models will inspire those charged with training and professional development to consider new and innovative strategies to increase skill and practice with delivery of EBP and EBTs. Increasing the dexterity of the mental health workforce in applying these methods will ultimately enhance clinical outcomes. 


\section{References}

Association of Directors of Psychology Training Clinics (ADPTC) Practicum Competencies Workgroup. (2004). Report on practicum competencies. Retrieved 10/10/2011 from:

http://www.aptc.org/public_files/Practicum\%20Competencies\%20FINAL\%20(Oct\%20'06\%20 Version).pdf.

Baird, B. (2007). Internship, practicum, and field placement handbook: A guide for the helping professions. New York: Pearson.

Beidas, R. S. \& Kendall, P. C. (2010). Training therapists in evidence-based practice: A critical review of studies from a systems-contextual perspective. Clinical Psychology: Science and practice, $17,1-30$.

Conner-Smith, J. K. \& Weisz, J. R. (2003). Applying treatment outcome research in clinical practice: Techniques for adapting interventions to the real world. Child and Adolescent Mental Health, 8, 3-10.

Essock, S. M., Goldman, H. H., Van Tosh, L., Anthony, W. A., Appell, C.R., Bond, G. R.,...Drake, R.E. (2003). Evidence based practices: Setting the contenxt and responding to the concerns. Psychiatric Clinics of North America, 26, 919-938.

Fristad, M.A., Goldberg-Arnold, J.S., \& Gavazzi, S.M. (2002). Multifamily psychoeducation groups (MFPG) for families of children with bipolar disorder. Bipolar Disorders, 4, 254-262.

Fristad, M.A., Verducci, J.S., Walters, K., \& Young, M.E. (2009). Impact of multifamily psychoeducational psychotherapy in treating children aged 8 to 12 years with mood disorders. Archives of General Psychiatry, 66, 1013 -1021. 
Gotham, H.J. (2006). Advancing the implementation of evidence-based practice into clinical practice: How do we get there from here? Professional Psychology: Research and Practice, 37, 606-613.

Herschell, A. D., Kolko, D. J., Baumann, B. L., \& Davis, A. C. (2010). The role of therapist training in the implementation of psychosocial treatments: A review and critique with recommendations. Clinical Psychology Review, 30, 448-466.

Hunsley, J., \& Lee, C. M. (2007). Research-informed benchmarks for psychological treatments: Efficacy studies, effectiveness studies, and beyond. Professional Psychology: Research and Practice, 38, 21-23.

Hyde, P.S., Falls, K., Morris, J. A., \& Schoenwald, S.K. (2003). Turning Knowledge into Practice: A Manual For Behavioral Health Administrators and Practitioners About Understanding and Implementing Evidence-Based Practices. Boston: Technical Assistance Collaborative, Inc.

Institute of Medicine. (2001). Crossing the quality chasm: A new health system for the $21^{\text {st }}$ Century. Washington, DC: Author.

Kendall, P.C., Gosch, E., Furr, J.M., \& Sood, E. (2008). Flexibility with fidelity. Journal of the American Academy of Child and Adolescent Psychiatry, 47, 987-993.

Lehman, A.F., Goldman, H.H., Dixon, L.B., \& Churchill, R. (2004). Evidence-based mental health treatments and services: Examples to inform public policy. New York, NY: Milbank Memorial Fund.

MacPherson, H. A., Leffler, J. M., \& Fristad, M. A. (2012). Implementation of Multi-Family Psychoeducational Psychotherapy (MF-PEP) for Childhood Mood Disorders in an Outpatient Community Setting. Manuscript submitted for publication. 
Stewart, R.E., \& Chambless, D.L. (2007) Does psychotherapy research inform treatment decision in private practice? Journal of Clinical Psychology, 63, 267-281.

Rakovshik, S. G., \& McManus, F. (2010). Establishing evidence-based training in cognitive behavioral therapy: A review of current empirical findings and theoretical guidance. Clinical Psychology Review, 30, 496-516.

Stirman, S.W., Bhar, S.S., Spokas, M., Brown, G.K., Creed, T. A., Perivoliotis, D., ...Beck, A.T. (2010). Training and consultation in evidence-based psychosocial treatments in public mental health settings: The ACCESS model. Professional Psychology: Research and Practice, 41,4856.

Weissman, M. M., Verdeli, H., Gameroff, M. J., Bledsoe, S. E., Betts, K., Mufson, L., ... Wickramaratne, P. (2006). National survey of psychotherapy training in psychiatry, psychology, and social work. Archives of General Psychiatry, 63,925-934.

West, A.E., \& Pavuluri, M.N. (2009). Psychosocial treatments for childhood and adolescent bipolar disorder. Child and Adolescent Psychiatric Clinics of North America, 18, 471-482.

West, A.E., Jacobs, R.H., Westerholm, R., Lee, A., Carbray, J., Heidenreich, J., \& Pavuluri, M.N. (2009). Child and family-focused cognitive-behavioral therapy for pediatric bipolar disorder: Pilot study of group treatment format. Journal of the Canadian Academy of Child and Adolescent Psychiatry, 18, 239-246. 
Table 1: Clinical/Clinical Research Rotation in PMDC

\begin{tabular}{l|l} 
Clinical Practice (10\%) & Clinical Research (10\%)
\end{tabular}

- Conduct intake evaluations

- Training in CFF-CBT/RAINBOW manual

- Carry caseload of 3 clinic patients

- Deliver manual-based treatment to 2

- Serve as co-facilitator of RAINBOW parent participants in RCT group

- Develop research project evaluating role of

- Clinical supervision parent psychopathology in predicting treatment outcome; data analysis and writeup 
Table 2:Detailed MF-PEP training approach

Content

Overview of psychoeducation psychotherapy as a model of care

Overview of treatment manuals

Outline of session format/structure

Review Child group therapeutic exercises

Review Parent/family group therapeutic exercises

Overview of research protocol and aims

Discussion of potential client population and screening procedures

Use of consultation/supervision

Review video clips of sessions

Wrap up Q \& A

Notes: all time is recorded in minutes; $*=$ two hours were spent on either the Child or the

\begin{tabular}{ccc}
$\begin{array}{c}\text { Original } \\
\text { Training } \\
(5 \text { hrs. })\end{array}$ & $\begin{array}{c}\text { Broad } \\
\text { Training } \\
(7 \text { hrs. })\end{array}$ & $\begin{array}{c}\text { Focused } \\
\text { Training } \\
(4 \text { hrs. })\end{array}$ \\
30 & 30 & 30 \\
20 & 15 & 15 \\
10 & 15 & 15 \\
60 & 120 & $120^{*}$ \\
60 & 90 & $120^{*}$ \\
N/P & 60 & 15 \\
15 & 60 & 15 \\
& & \\
N/P & 15 & 15 \\
60 & N/P & N/P \\
45 & 15 & 15 \\
\hline
\end{tabular}

hrs.

$7 \mathrm{hrs}$.)

hrs.)

30

15

$60 \quad 120 \quad 120 *$
Parent/family exercise were trained; $N / P=$ not present 
Table 3: Response to Training Experience

\begin{tabular}{|c|c|c|c|c|c|c|}
\hline \multirow[t]{4}{*}{ Item } & \multicolumn{6}{|c|}{ Method } \\
\hline & \multicolumn{2}{|c|}{ Original } & \multicolumn{2}{|c|}{ Broad } & \multicolumn{2}{|c|}{ Focused } \\
\hline & M & $\%$ Yes & M & $\%$ & M & $\%$ Yes \\
\hline & & & & Yes & & \\
\hline Did training prepare you to run the group & 1.17 & 83 & 1.00 & 100 & 1.50 & 50 \\
\hline $\begin{array}{l}\text { Were there topics/skills you did not get } \\
\text { enough training on }\end{array}$ & 1.83 & 17 & 2.00 & 0 & 1.50 & 50 \\
\hline $\begin{array}{l}\text { Have you been trained on other } \\
\text { manualized interventions }\end{array}$ & 1.67 & 33 & 1.00 & 100 & 1.25 & 75 \\
\hline
\end{tabular}

